\title{
Revista de Revistas
}

\section{Veinte años de Ginecología Funcional} en la cátedra de Ginecología.

Doctor Walter Kock - Boletín de la Sociedad Chilena de Obstetricia y Ginecología $N^{\circ}$ 8. Noviembre 1952.

El autor manifiesta que basado en su experiencia ha verificado conocimientos y ha rectificado pensamientos. Hace estas observaciones importantes.

Hasta hace poco, según Schroeder, se asignaba a los ovarios tres funciones especificas: generativa, vegetativa y somática. Sin embargo los ovarios ejercen sólo dos funciones conocidas: función enodcrina o humoral y función exocrina o gameto genética.

Insiste en la importancia de aceptar el concepto de autoregulación entre los ovarios, la hipófisis anterior $y$ los órganos efectores de las hormonas sexuales para que el clínico pueda hallar bases seguras en el diagnóstico. pronóstico y tratamiento. Anota que el metabolismo basal es un auxiliar de exploración funcional tiroldea para ol endocrinólogo experimentado; confirma el diagnóstico cuando coincide. pero no lo invalida cuando discrepa; presta utilidad para controlar y verificar los efectos de los fármacos quo se apliquen. Considera que la Somatometría, menospreciada por muchos ginecólogos, es un importante y exac- to medio de exploración endocrina. En cuanto a la Colpocitología, manifiesta que ha declinado el optimismo por este examen; es un medio útil si está en manos de un ginecólogo que sepa interpretarlo correctamente. Aclara la importancia de la radiografía de la silla turca citando un estudio de más de 3.000 placas. Opina que la temperatura de base no es tan útil, como otros lo indican; creé que hay un factor psiquico que influye en el desenvolvimiento de las curvas. Termina estudiando los sindromes endocrino-genitales como debidos a alteraciones de uno de los tres elementos que intervienen en el mecanismo de función del aparato genital: ovarios, hipólisis - efectores de las hormonas ováricas.

\section{Analgesia obstétrica}

Doctores Alberto Larenas, Osualdo Varas y Juan Ferrari. Boletin de la Sociedad Chilena de Obstetricia y Ginecología. Vol. XVII. Octubre del 52 No 7.

Los autores hacen en extenso y meritorio estudio en 2.222 parturientas. Los métodos empleados varian según el estado de la paciente al ingresar $C$ la clínica, distingue tres grupos: $1^{\circ} \mathrm{Pa}-$ 
cientes en el período final del trabajo. - Se les aplicó ciclopropano, protóxido de nitrógeno, anestesia raquídea tipo - saddle block, con Procaína, Pantocaína o Nupercaina; Analgesia endovenosa sola o complementada con Hoque pudendo. Anotan el peltgro de : Ahrilatón ventricular durmte la malgesta con ciclonropano si se aplian extractos retrohipotisarios por vía ndovenosa. $2^{\circ}$ Pacientes que ingresan a mediados del periodo de dilatación. Emplean el Demerol como analgésico y antiespasmódico; la Escopolamina como amnésico y la Apomorfina como sedante y antiemético. Han empleado el Clorhidrato de Metadona en reemplazo del Demerol, con resulados muy inferiores, por lo cual lo desaconseian. $3^{\circ}$ Pacientes que ingrescm al comienzo del trabajo. Aplican rimero barbitúricos de acción corta 0 moderada (Seconal, Amital Sódico); luégo, obtenida una dinámica satis:actoria, continúan como en el segundo grupo.

No conctben que en la actualidad la raciente no sea analgesiada durante

I parto por alguno de los métodos coacidos de comprobada seguridad mito para la madre como para el hilo. Dicha seguridad sólo se consigue si la: línica cuerita con un equipo completo de tocólogos, anestesistas y pediatras experimentados. Concluyen demostrando que la paciente analgesiada es la que más requiere una conducta activa por parte del obstetra para que controle cuidadosamente el pulso, 1. tensión arterial la respiración, la dinámica uterina, grado de dilatación cervical y carácter de los ruldos fetalos, todo lo cual es anotado gráflcamente en una ficha especial. Debido a este riguroso control comprueban que la analgesia no interfiere con la buena marcha del trabajo ni que se prosenten accidentes con mayor frecuencia que en los partos no sometidos a ningún método analgésico.

\section{Hipertensión cuterial y embarazo.}

Doctor Elías Cumsille Núñez. Boletín de la Sociedad Chilena de Obstetricia y Ginecología. Vol. XVII. Diciembre, 1952. No 9.

El autor después de analizar las causas de la hipertensión en general, concluye que la hipertensión gravídica sería un reflejo compensador, cuando el útero gestante requiere urgentemente más sangre para garantizar la vida del feto. Como consecuencia de muchas experiencias, de los hallazgos hormonales en la toxemia, de la anatomía patológica de la placenta eclámHica, formula una teoría que él considera aliamente sugestiva, aun cuando tenga puntos débiles que reparar. - Las modificaciones toxémicas (alza de las gónadotropinas coriónicas, descenso en estrógenos $y$ progesterona) son idénticas a las que preceden al parto normal, debidas al envejecimiento de la placenta. Envejecimiento lógico en el parto normal, prematuro en el caso de toxemias. La causa de este envejecimiento pudiera ser debido a un insuficiente aporte de sangre ante el cual la placenta alteraria su producción hormonal, liberando al mismo tiempo a la circulación general, una sustancia vasopresora con el fin de forzar su riego sanguíneo; por 
- tro lado cabría la pusibilidad que la decidua, por el desequilibrio hormonal produjese una sustancia de acción tćxica vascular". En cuanto al tratamiento plantea medidas usuales unánimemente aceptadas, tan: solo que al mokr la necesidad de disminuir las Mturs animales en la dieta cons:der que éstas no deben eliminars totalmente mientras no exista hipertensión con retención uréica ya que de ?o contrario el efecto seriu perjudicial para la madre y el fetc.

\section{Segundo caso colombiano de toxoplasmosis congénita.}

Doctores Alfredo Laverde y luan Arciniegas C. Anales de lo Sociedad de Biología de Bogotá. Vol. 5. Mayo del $53, N^{\circ} 6$.

Anima a los autores, al presentar este caso, la muy laudable intención de señalar tanto a los pediatras como a los tocólogos, la confirmación por sequnda vez de la existencia de la toxplemosis congérita en nuestro país. El nimer caso fue descrito por los doctores Manuel Roca García, Jorge Camacho Gamba y Gonzalo Esguerra Gómez. Fue presentado a la Academia Nacional de Medicina en 1950 y publicudo en la Revista Colombiana de Pediatría y Puericultura, No 5 , año $X$, página 238. Anotan que la Toxcplasmosis, ocasionada por el Toxoplrsma Gundii, $\in 3$ una entermedad crei siempre mortal, que ataca sobre to to al recién nacido y que consisto on und encefalomielitis con-complicaclones oculares $y$ en particular corioretinitis: aparecen calcificaciones in- tracraneanas particulares, como testigo de la encelalitis fetal. El caso por ellos presentado tiene como interés primordial la supervivencia del pacients y algunos hallazgos radiológicos no señalados en la literatura.

\section{Cambio cualitativo producido por la radiación en las miltosis del cáncer de cuello de útero}

Por el doctor Eugenio Recasens Méndez-Queipo de Llano, Médico interno del Servicio Maternólogo del Estado.

Este estudio lo hicimos uasándonos en la idea de Glucksmann (biopsias seriadas antes y después de la radiación), de tal manera, que tomábamos una biopsia, rplicábamos radio y comparábamos la biopsia a que antes nos hemos referido corı una nueva tomada después de la radiación: Hacíamos hincapié en la necesidad de buscar para este estudio porciones de teiido joven, activo (las zonas necróticas. como es natural, no sirveri).

Vimos entonces que indudablemente uno de los primeros efectos de la radiación es la inhibición mitósica de las células. Que esta inhibición tenga lugar por una lesión de impacto sobre los cromosomas, producida por ia radiación (Mueller y Stadler) o bien que, como quiere Dale, la acción de la radiación tenga lugar sobre los enzimas intermoleculares, es algo que ni cuando hicimos el trabajo a que ya nos hemos referido, ni cuando escribimos $e$ l presente, estamos en condiciones de discutir. 
El hecho, sin embargo, es exacto y los sirve para juzgar la radiosensibililad de un tejido. Después de la radiaión, la escasez e incluso la talta de nitosis es cierta y ello nos explica la endencia a envejecer de la colonia velular, pues las células jóvenes (céwh en reposo de Glucksmann) tienen in general que diferencicrse 0 degnierar, ya que su poder de división ha sido anulado. En un caso $c$ en otro el esultado final será el mismo: dismiuución del ingrediente joven de la coonia.

También describiamos entonces el sfecto indirecto de la radiación sobre a colonia celular cancerosa, que disninuyendo el riego sanguíneo, produziendo una reacción inflamatoria, una reacción conjuntiva, etcétera, contribuía también a la maduraclón o envejecimiento de la colonia celular. Y esto porque las condiciones adversas que. como hemos visto, crea, se manifieslan primero en las zonas del tumor que están en plena actividad, en las zonas jóvenes.

También haciamos referencla a otro fecto que producido por la radiación. encontrábamos en nuestro estudio: la lendencia de los tejidos irrajiados que forman el tumor, a normalizar sus esructuras. El tejido conjuntivo reaccioha en general después de la radiación defensivamente, mostrando una neogénesis tibroblástica intensa, que es causa de un aumento de la resistencia a la invasión neoplásica. El telido eplelial tiende a organizar normalmente su arquitectura. Este hecho se traduce unas veces en la formación de una pseudo-basal, otras en el establectmiento de relaciones entre sus células. expresadas éstas por la aparición de espinas intercelulares, etc.

Pues bien, usi como la inhibición mitósica disminuyendo el número de mitosis, favorece la normalización de la estructura del tejido canceroso irradiado, también la disminución de ciertas formas mitósicas (metafísicas) y el aumento en cambio de otras (pro ana y telelásicas), hace más real la normalización del tejido epitelial a que nos estamos refiriendo.

Esta variación cualitativa de las mitosis en el carcinoma de cuello irradiado. constituye el objeto del presente trabaio.

Queremos anle todo reseñar brevemente, mediante un cuadro sinóptico que tomamos del prolesor Ramb́ y Cajal, las fases que tiene el proceso mitósico normal; claslficación ésta que ha servido como base en nuestras abservaclones.

La mitosis normal atraviesa sucesivamente por una serie de fases, pro. gresivas primero y regresivas luégo. al tinal de las cuales nos vamos a encontrar con dos célulis hijas.

Son progresiva: todas las fases que atraviesa la célula que entra en milosis hasia llegar a la nuetakinesis o lase de estrella madre y regresivas las que median entre esta lase de-metakinesis y el estado de descanso o reposo de las células hilas. 
Fases progresivas

Faser regresivas

$$
\begin{aligned}
& \left(\text { Profase } \ldots \ldots \ldots \ldots \mid \begin{array}{l}
\text { Fase de descanso. } \\
\text { Fase de reticulación fina. } \\
\text { Fase de reticulación gruesa. } \\
\text { Fase de ovillo. }
\end{array}\right. \\
& \text { Metalase ....... } / \begin{array}{l}
\text { Fase segment. transversal. } \\
\text { Fase de estrella madre. }
\end{array} \\
& \text { I Fase segment. longitudinal. } \\
& \text { | Anatase ....... } \begin{array}{l}
\text { Fase d placa ecuatorial. } \\
\text { Fase de estrellas hijas. }
\end{array} \\
& \text { Teletase ....... }\left\{\begin{array}{l}
\text { Fase de ovillo hijo. } \\
\text { Fase de reticulación. } \\
\text { Fase de descanso. }
\end{array}\right.
\end{aligned}
$$

Nosotros hemos empleado parr nuestro estudio la coloración de Feulgen, especifica, según la mayor parte de los autores para el ácido desoxiribonucleico, que está íntimamente ligado a la cromatina nuclear. Con ella se obtienen preparaciones en que los núcleos colulares resalton muy claramente (véase microfotografia número 1).

Todo célula contiene en su núcleo dos tipos de ácidos nucleicos, el ya mencionado desoxiribonucleico (ADN), que, como ya hemos dicho, parece que no se encuentra en las células de los tejidos más que en la cromatina nuclear, y el ácidp ribonucleico (ARN). que abunda en el eitoplasma $y$ en el nucléolo y que existe también, en pequeñas cantidades, en la cromatina. Estas dos sustancias son polinucleótidos polimerizados, estondo compuesto cada unidad de los mismos, o nuclétido, por tres elementos: una pentosa, una base purínica o pirimidínica $y$ un radical ortofosfórica. La polimerización de estos nucleótidos se logra por una unión de tipo estérico de cada elemento ortofosfórico con el azúcar del siguiente nucleótido:
La diferencia que existe entre $\epsilon$ l $A D N$ y el ARN es que en este último ei grupo desoxipentosa está ocupado por un grupo pentosa.

Lo que para nuestro estudio resulta interesante es que el ácido desoxir:bonucleico es sintetizado en gran cantidad por la célula cuando comienza la mitosis de la misma, mientras que el ribonucleico tiende a desaparecer en esta época de la vida celular (muchos bioquímicos opinan que esta disminución del ribonucleico tiene lugar por la transformación de! mismo en ácido desoxiribonucleico). Luégo, con la aparición de las nuevas proteínas endocelulares aparece, en gran cantidad, el ácido ribonucleico nuevamente, pues debe estar en relación con la neogénesis protelca.

Lo cierto es que al alcanzar la célula su momento de reproducción, el núcleo se encuentra grandemente cargado de ADN y se tiñe perfectamente con el método de Feulgen, en tanto que el de las células en reposo. aunque también teñidos, ofrecen muchn menor contraste. Esta diferencia aumenta al disminuír la iluminación (vẻase microlotogralia número 2). 
Dicho esto, a modo de introducción - y de aclaración a nuestro trabajo, vamos a reseñar brevemente el fundamento y la técnica de la coloración de

\section{Foulgen.}

En el método se comienza por producir una hidrolisis ácida de la preparación histológica, que tiene como consectencia la descomposición ráp:da de los cuerpos purínicos del núcleo. surgiendo en consecuencia una serie de grupos aldehídicos reductores, que son los que al combinarse con el ácido sulfofucsínico forman una sustancia rojo-violeta intensa. En el protoplasma celular no existen grupos aldahídicos, y, por tanto, la coloración no tiene esta parte de la célula.

Se debe comenzar por dejar los cortes durante veinticuatro horas en alcohol de $96^{\circ}$ al que se añads un $1 \%$ de dimethylciklohezndion (dimedon, Kahlbaum) para que se combine con los aldehidos existentes, inutiliźndolos para la reacción siguiente.

Hidrolisis-Inmersión duranto un par de minutos en $1 / 1 \mathrm{n}$. ácido clorhidrico. Después se llevan a un recipiente que contiene el mismo ácido clcrhídrico a la temperatura exacta de $60^{\circ}$ (se hac : bien al baño de maría). Aquí se dejarán cuatro minutos, durante los cuales tiene lugar la hidrolisis les importante ajustarse bien a la temperatura $y$ al tiempo que hemos dicho). Luego se interrumpe la hiaroilisis ilevando los cortes nuevamente al clorhídrico frío durante breve rato, lavándolos luégo abundantemente en agua destilada.

Coloración-1-1'30 horas en ácido sulfofucsínico. Luégo lavado en agua que contiene $\mathrm{SO}_{2}$. Lavado en aqua abundante, secado, alcohol, montain con bálsamo, etc.
Preparación de las soluciones:

Acido clorhidrico. $82,5 \mathrm{~cm}^{3}$. de CLH (peso específico l'19) en 1.000 gramos de agua destilada.

Acido sufofucsinico 1 gramo do Fucsina (parafucsina) en polvo, so mezcla con $200 \mathrm{~cm}^{3}$. de agua hirviente. Se agita para favorecer la solución $y$ se deja ir enfriando lentamente. Cuando llega a $50^{\circ}$, filtrado a un frasco con tapón esmerilado y se cañaden $20 \mathrm{~cm}^{3}$. de $\mathrm{n}$. CLH. Se acaba de entriar (temperatura ambiente) y se anade un gramo de bisulfito sódico pro analysi. Se deja reposar $24 \circ 48$ horas, durante las cuales debe decolorarse el líquido. que se conserva bien en la oscuridad y bien tapado. En cuanto se colorea hay que renovarlo.

Líquido gue contiene $S O .200 \mathrm{~cm}^{3}$. de agua destilada a los que se añade:ı $10 \mathrm{~cm}^{3}$. de solución acuosa saturada de bisulfato sódico. Luégo de mezclar bien se añaden $10 \mathrm{~cm}^{3}$. de clorhídrico normal. Este líquido se oxida on contacto con el aire y, por lo tanto, hay que conservarlo bien cerrado (para. fina). Debe oler fuartemente a $\mathrm{SO}_{2}$.

Con este líquido se llenarán tres vasos y los cortes se irán pasando de upo al otro, dejándolos cada vez l'30 minutos. Luégo lavado con agua destilada.

\section{Cambio cualitativo de las mitosis en el carclnoma de útero radiedo.}

Si contamos las mitosis que encontramos en un tejido normal, veremos que la mayor parte de las mismas se encuentran en profase, anafase o tolefase, y sólo muy pocas en metafase. 
Nesotros hemos estudicudo 83 biop- as de útero (carcinoma cervical) y ics hemos encontrado que en ellas curre exactamente lo contrario. Y es esto que el mayor número de mitosis que hemos encontradu estaban en meutase y solamente muy pocas en protase anafase o telefase. En esto coindimes con los trabaios de P. C. BöLer $y$ otros investigadores.

Sumando el número total de mitosis que hemos contado y hallando los tantos por ciento de metáases, profases, anafases y telefases que hemos visto, llegamos a los siguientes resultados:

$\%$

élulas en metafase $\ldots \ldots \ldots \ldots$. 61

Celulas en profase.......... 28

Celulas en anatase............ 3

Células en telefase ............ ?

En general, como ya hemos dicho, coincidimos con las experiencias de otros autores, perc hemos-de hacer constar que las células en profase eran mucho más abundantes que las en - anafase $y$ telefase juntas, que alcanzaban sumadas solamente un $11 \%$ ( $28 \%$ de células en profase). P. C. Kö. !er da como sensibleme te iguales of numero de células en profase al do analase y telefase simadas.

Siguiendo la técnica a que hemos hecho referencia al principio del presente trabajo, observamos que después de la radiación ta reducción procentual de célu'as en mitosis, se hace principalmente a base de las células en metafaso: de tal manera, que - podemos decir, que después de la radiación aparte de la reducción total del tanto por ciento de células en mi- tosis, se observa una dismil ución marcada de las células en metafase, lo que hace que los respectivos tantos por ciento de células en pro, meta, ana $\mathrm{y}$ telefase se hagan sensiblemente iguales, no predominando ya las formas metafásicas y asemejúndose con ello a la proporción que es fisiológica ! teiido normal.

Esta variación cualitative de las colulas mitósicas después de la radiación, cuya comprnbación constituye. ya lo hemos dicho, el objeto del presente trabajo, corrobora en cierto modo, lo que decíamos antes: que el carcinoma irradiado tiende a normalizar la arquitectura propia del tojido originario.

\section{Carcinoma del cérvix en tase de preinvasión durante el embarazo}

Se acepta casi universalmente que el embarazo determina camblos específicos en el epitelio del cérvix. y cas! de un modo tan extendido se admito que pueden acaecer clertos acambios. que a veces se confunden con el carcinoma preinvasor del cérvix. Por esto motivo, muchos autores estiman que no puede ni debería hacerse el diagnóstico del carcinoma preinvasor del cérvix en pacientes embarazadas. Epperson y sus colaboradores han indicado que las lesiones diagnosticadas en la fase preinvasora del carclnoma del cérvix durante la gravidez desaparecieron espontánemente al terminar el embarazo, deducléndose de ello que los observados oran cambios gravídicos, y no un verdadero carcinoma prelnvasor. 
Sin embargo, Greene y sus colabo: radores no aceptan que el embarazo cause cambios especificos en el epitelio cervical, aun reconociendo que las metaplasias e hiperplasias de los elementos glandulares son más frecuen. tes y prominentes durante el embarazo que antes o después del mismo, sin ser cambios típicos de la gravidez. También aceptan que el epitelio escamoso estratificado superficial del cérvix crece rápidamente durante el embarazo y que orasionalmente se observa un leve aumento en el espesor del estrato de células basales con núcleos normales. Asimismo, admiten que cuando existen los signos indicadores de un diagnóstico de carcinoma preinvasor, debe hacerse dicho diagnóstico esté o no emburazada la paciente. Los autores estiman que esta controversia sobre el carcinoma preinvasor en pacientes embarazadas sólo puede dilucidarse por el estudio continuado de las pacientes que presentan tales lesiones, y determinando si desaparecen - no con la terminación del embarazo. Los datos presintados por los autores se han obtenido de un estudio de este tipo.

Se diagnosticó un careinoma preinvasor del cérvix en las biopslas recilzadas en 14 pacientes embarazadas. observadas durante el embarazo $y$ puerperio. La les'ón persistió después de la terminación del embarazo en i2 de las 14 pacientes, hallándose en las biopsias, on las piezas operatorias 0 en ambas. Los autores destacan que. con dos excepciones, estas lesiones - se tratara o no de un verdadero carcinoma - no fueron a cambios gravidicoss, y que en vez de desmarecer. persistieron después de termmar of embarazo. En 2 de los 14 casos los al1tores no hallaron ningund anormal:dad post-parto. La neces:dad de establecer una prueba viene a recaer en el clínico que afirme que la lesión s? extirpó totalment- en la biopsia o je destruyó por cambios inflamatorios, $y$ por este motivo no se volvió a hallar otra vez. Los autores no hacen una afirmación similar. Es posible que la le. sión no fuera un verdadero carcinom? preinvasor, sitho un cambio reversib!s En cada una de estas pacientes se han practicado 4 biopsias en los cuadrantes uterinos dos veces post-parto. Estas paciantes se seguirán estudiando ulteriormente. En una de estas pacientes el frotis fue positivo en el mamento de hacer la primera biopsia postparto (17 semanas), siendo negativa al cabo de 31 semanas.

(R. R Grecne, B. M. Peckham I. T. Chung, y otros del Deparlamento d Obstetricia y Ginecología de la Northwestern University Medical School $y$ del Wesley Memorial Hospital y del Chicago Maternity Center, Chicago Illinois: Surgery, Gynocology and Obs-

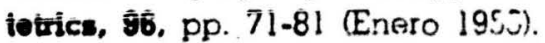

